\title{
La Classe di Bayes: note metodologiche, epistemologiche ed operative per una reale digitalizzazione della didattica nella scuola italiana*
}

\section{Paolo Ferri - Stefano Moriggi}

Università degli Studi Milano-Bicocca, Dipartimento di Scienze Umane per la Formazione doi: 10.7358/ecps-2014-010-ferr

paolo.ferri@unimib.it stef.moriggi@gmail.com

BAYES' CLASSROOM: METHODOLOGICAL, EPISTEMOLOGICAL AND OPERATIONAL NOTES

FOR A REAL DIGITALIZATION OF TEACHING IN THE ITALIAN SCHOOL SYSTEM

\section{Abstract}

The gap between the new "digital natives» learning styles and teaching strategies and practices - still very traditional in Italy - is wide. We need to radically re-think the teaching and learning setting, at least, with regard to three fundamental issues: (a) the physical spaces of education; (b) the methodological approaches; (c) the technologies involved in the learning process. The aim is to sketch a possible design for the new digitally augmented education, based on a social-constructivist approach. The analysis of data and evidence related to behavioral and cognitive styles of the younger generations have led us to identify the logic of scientific discovery (in this case, understood in terms of Bayesian epistemology) as the best suited cultural matrix to develop a model of augmented education. In order to illustrate our proposal, we first describe how and why the brain-frame of "digital natives» is different when compared to our Gutenberg-based way of teaching. The second part describes the design of our methodological and technological proposal, and outlines its three step-struc-

S. Moriggi.

* I parr. 1 e 2 del presente articolo sono opera di P. Ferri, i parr. 3 e 4 sono opera di 
ture: tool box, cooperative problem-solving, and situation room. The third part provides some remarks on the impact of this approach on the concept of democratic citizenship.

Keywords: Bayesian epistemology, Didactics, Digitalization, Digital natives learning styles, Italian school.

\section{COME STANNO CAMBIANDO GLI STUDENTI: I «NATIVI DIGITALI» E LA TRASFORMAZIONE DELLA DIDATTICA}

Negli ultimi due decenni si sono affacciati alla scuola generazioni di studenti noti come "nativi digitali» (Prensky, 2001, 2006 e 2012; Gasser \& Palfrey, 2008; Ferri, 2010, 2011 e 2014; Livingston et al., 2011). Si tratta di coorti di bambini e pre-adolescenti nati e cresciuti in ambienti e contesti corredati di schermi interattivi ${ }^{1}$. Come già emerge in una pionieristica indagine del BECTA nel 2008, (Smith, Rudd, \& Coghlan, 2008) ${ }^{2}:$ «[... considerati nel loro insieme, gli effetti combinati della rivoluzione digitale e del Web 2.0 sui sistemi educativi si concretizzano in una radicale trasformazione che tende a riorientare i sistemi di istruzione/apprendimento verso una struttura didattica che privilegia un approccio centrato sullo studente» (Crook, 2008). In particolare, le grandi aree di impatto dell'innovazione prodotta dalla transizione al digitale e dal web 2.0 sulle pratiche di apprendimento e sulle forme di condivisione e creazione del sapere tra gli studenti possono essere riassunti come segue:

a. una sensibile crescita dei comportamenti di ricerca/esplorazione nell'apprendimento, rispetto ai comportamenti acquisitivi e passivi di ricezione dei contenuti;

b. una spiccata fluency tecnologica degli studenti per i quali il web diventa il medium primario di ricerca, di acquisizione e di condivisione dei contenuti del sapere;

c. un netto incremento dei comportamenti di collaborazione/cooperazione tra pari, messi in atto in particolare attraverso strumenti quali Facebook, YouTube o altri social network (Jenkins, 2009);

1 Nella ricerca sviluppata con il finanziamento della J. Walter Thompson, per esempio, sono stati definiti la Generation Z (J.W.T., 2012).

2 La ricerca è stata condotta presso il BECTA, il prestigioso istituto di ricerca inglese sull'innovazione didattica ora chiuso a seguito di un provvedimento del Primo Ministro conservatore David Cameron. 
d. una rilevante tendenza da parte degli studenti a privilegiare l'espressione della propria identità e delle proprie idee attraverso social network, strumenti di messaggistica istantanea, ancora più che blog o microblogging.

Stanno, infatti, emergendo nuovi stili cognitivi che sono il portato della reinterpretazione da parte dei nativi digitali della rivoluzione tecnosociale prodotta dal «capitalismo informazionale» (Castells, 1996). E il sociocostruttivismo pedagogico risulta essere una prospettiva teorica efficace per progettare e gestire una transizione consapevole verso modelli didattica aumentati dalle tecnologie.

Infatti, le pratiche di comunicazione e di interazione online proprie del web 2.0 sembrano ben armonizzarsi con le pratiche pedagogiche dell'attivismo e del learning by doing. E i «nativi», attraverso il loro «stile di apprendimento digitale», incarnano e rappresentano un nuovo orizzonte entro cui modellare una didattica compatibile alle loro prerogative cognitive e relazionali.

A fronte di una richiesta sempre maggiore di autonomia e di una tendenza alla costruzione (oltre che alla condivisione) con i pari delle forme e dei risultati del loro apprendimento, si delinea pertanto l'opportunità di prediligere un approccio tale da consentire una sperimentazione diretta della cosiddetta pedagogia dell'errore, ma calata in strategie e logiche strutturalmente orientate al trial and error.

Sono proprio la condivisione tra pari, la cooperazione, la ricerca di differenti approcci al problema dato e di molteplici codici e piani di interpretazione (e di rappresentazione) utili alla risoluzione del medesimo gli atteggiamenti determinanti che marcano le loro differenze rispetto agli immigranti digitali. Pertanto, è proprio in questo contesto che, secondo le regole comuni all'etica hacker ${ }^{3}$ e alla nuova cultura partecipativa dei nativi - condivisione, gratuità, cooperazione - (Castells \& Himanen, 2002; Jenkins, 2009), va de-

3 I principi generali su cui si basa l'etica hacker sono: condivisione, apertura, decentralizzazione, libero accesso alle tecnologie informatiche, miglioramento del mondo. Secondo Himanen, inoltre, «l'etica hacker è una nuova morale che sfida l'etica protestante e lo spirito del capitalismo che si fonda invece su «la laboriosità diligente, l'accettazione della routine, il valore del denaro e la preoccupazione per il risultato». In confronto alla morale presentata da Weber, l'etica hacker si basa piuttosto sul valore della creatività e consiste più precisamente nel combinare la passione con la libertà. Il denaro cessa di essere un valore di per sé e il beneficio si misura in risultati come il valore sociale e l'accesso libero, la trasparenza e la franchezza. Nell'opinione di Himanen, l'etica hacker è molto vicina all'etica della virtù descritta da Platone e da Aristotele. Gli hacker, condividendo risorse e formando community in cui si verifichi un continuo e costante scambio di informazioni e insegnamenti, recuperano valori tipici della società moderna europea, dando nuovo vigore all'etica della cittadinanza. In tale prospettiva riveste un ruolo centrale il computer, che «accresce la libertà di espressione e della creatività» (Himanen, 2001). 
finendosi un inedito universo di pratiche e di significati che noi abbiamo il compito di riconoscere, analizzare e gestire ${ }^{4}$.

Pare evidente, alla luce di quanto fin qui osservato, che per colmare il gap tra i nuovi stili di apprendimento dei giovani e le strategie insegnamento, ancora molto tradizionali e improntate al puro trasferimento di conoscenze, sia necessario un nuovo modello didattico.

Questo modello che proveremo ad abbozzare nei paragrafi seguenti ha due obiettivi.

Anzitutto, inquadrare (anche epistemologicamente) una trasformazione della didattica in evoluzione che non riguarda solo la scuola, ma tutta la società - in particolare quella europea.

Basterebbe, infatti, tener conto degli obiettivi indicati dalla Strategia di Lisbona ${ }^{5}$, concordata da tutti gli Stati membri, per prendere atto del fatto che nel nostro Paese il sistema della formazione superiore e professionale long life nella sua totalità richiede un profondo ripensamento in senso neoattivista e socio-costruttivista per quanto concerne sia le metodologie, sia gli obiettivi formativi.

Il secondo obiettivo consiste invece nella proposta di un modello di cooperazione razionale abilitato dalle tecnologie digitali - la Classe di Bayes congruente con le strategie dell'Unione Europea rispetto alla convergenza dei sistemi educativi e dunque funzionale a creare, in maniera adeguata alle esigenze di ogni livello scolare, competenze disciplinari e trasversali in grado di promuovere forme avanzate di capacità di gestione di contenuti complessi e di cittadinanza democratica attiva.

4 Si vedano a questo proposito per gli aspetti teorici, i New Literacy Studies (Cope \& Kalantzis, 2000); l'approccio delle Multiliteracies nell'ambito della Technology Enhanced Education (Pullen \& Cole, 2009); la Convergence Theory di Jenkins (2006). Altri riferimenti possono essere le ricerche di Jonassen (2005) sul technology enhanced learning, i lavori di Spiro et al. (2006) sulla flessibilità cognitiva, sull'emergenza di un nuovo modello pull di condivisione delle informazioni e di uso delle risorse tecnologiche.

5 Gli obiettivi per l'educazione della Strategia di Lisbona sono consultabili al sito http:// www.indire.it/db/docsrv//PDF/raccomandazione_europea.pdf; sullo stato di attuazione degli obiettivi di Lisbona si veda il documento disponibile al sito http://ec.europa.eu/education/ policies/2010/doc/progressreport06.pdf. 


\section{LA CLASSE NON È L'AULA: NUOVE INTERAZIONI E INEDITE COLLABORAZIONI}

\subsection{Le condizioni di possibilità di un'innovazione necessaria}

Siamo convinti che non siano le tecnologie in sé a modificare i processi formativi, ma piuttosto le pratiche concrete e il lavoro degli insegnanti e degli alunni che riscrivono spazi e modi delle strategie di apprendimento (Moriggi \& Nicoletti, 2009; Moriggi, 2014), dentro e fuori la scuola. Proprio la riflessione sulle pratiche comunicative e informali dei nativi digitali (Ferri \& Mantovani, 2006 e 2008; Ferri, 2008, 2011, 2013 e 2014; Ferri, Mizzella, \& Scenini, 2009) permette di delineare un nuovo scenario concettuale e operativo rispetto ai processi di apprendimento in grado di colmare il gap tra gli stili di insegnamento enciclopedico/trasmissivi dei docenti e quelli «partecipativi» (Jenkins, 2009) degli allievi.

Le tecnologie digitali offrono, infatti, oggi per la prima volta, la possibilità di rompere gli schemi della scuola tradizionale di massa, aumentando (in potenza) anche il carattere egualitario e democratico dell'esperienza formativa.

Apprendere attraverso il fare e lo scoprire (Dewey, 1938) - facendo concrete esperienze e sensate riflessioni su queste dinamiche nonché revisioni razionali delle evidenze acquisite - potrebbe offrire infatti agli studenti e agli insegnanti digitalmente «abilitati» un approccio critico e tollerante all'acquisizione delle conoscenze. Si tratta però di modulare all'interno delle pratiche didattiche formali una metodologia di insegnamento improntata alla "logica della scoperta scientifica» - in modo da plasmare su tale base una strategia di apprendimento e un setting didattico al contempo cooperativo e capace di mettere in valore le conoscenze e le attitudini individuali.

Studiare diventa così una scoperta razionale condivisa. Approfondiremo nel par. 3 il nucleo epistemologico del nostro modello - e con esso l'evoluzione della stessa definizione di "gruppo in formazione». Per il momento ci concentriamo sulla analisi delle condizioni di possibilità di questa proposta. La concreta opportunità di una svolta in questa direzione è agevolata anche da quattro ordini di ragioni:

1. Accessibilità. Internet garantisce un accesso potenzialmente gratuito ed egualitario a un numero sempre più vasto di fonti della conoscenza.

2. Intelligenza sociale. Le tecnologie digitali «naturalmente» inducono a un metodo interattivo e partecipativo nell'accostarsi alla conoscenza (Point, click and share).

3. Condivisione. Gli alunni e gli studenti nativi digitali praticano spontaneamente fuori da scuola questo tipo di comportamenti attraverso social- 
network e strumenti di comunicazione istantanea cui accedono attraverso smartphone, tablet, notebook, console per video giochi.

4. Sostenibilità. I costi della infrastrutturazione tecnologica sono considerevolmente calati negli ultimi dieci anni e verosimilmente continueranno a scendere (Ferri, 2013).

Non è un caso, infatti, se paesi all'avanguardia nell'innovazione didattica attraverso le tecnologie - come quelli del Nord Europa e la Gran Bretagna (Smith, Rudd, \& Coghlan, 2008) - si sono infatti già mossi in questa direzione, articolando progetti di sistema che prevedono la riprogettazione degli spazi e delle infrastrutture scolastiche e/o formative in congruenza con gli obiettivi di Lisbona relativi alle competenze digitali per il long life learnning ${ }^{6}$. Sulla base di quanto affermato nel par. 1.1, non è certo un azzardo considerare il sistema scuola italiano un late comer. Tuttavia, essere gli «ultimi arrivati» talvolta può rivelarsi persino una opportunità. A condizione, però, di ipotizzare una linea di intervento pensata e coordinata allo scopo di accompagnare l'innovazione metodologica e tecnologica.

Procediamo, quindi, a presentare la tipologia di setting e di infrastrutture che consente di trasformare l'aula tradizionale in una Classe di Bayes ovvero di un setting didattico aumentato dalla tecnologia, ma soprattutto implementato da una matrice culturale - appunto, l'epistemologia bayesiana - utile a definire e plasmare gli stili e le pratiche di un apprendimento attivo e cooperativo, ottimizzando al contempo le opportunità offerte dalla tecnologia e contenendone i potenziali rischi.

\subsection{Il setting didattico: tempi e spazi della scuola digitalmente aumentata}

Secondo la nostra ipotesi, il tempo-scuola di insegnanti e studenti nell'era digitale risulterà suddiviso in tre tipologie di attività di insegnamento/apprendimento: (a) Tool box, (b) Problem solving cooperativo e (c) Situation room. Il tempo da dedicare a ciascuna attività è ovviamente flessibile in relazione agli argomenti trattati, alle necessità della programmazione e al contesto situato della classe. Tuttavia, nella nostra proposta il Problem solving cooperativo (e cioè la seconda delle fasi che di seguito descriveremo) dovrebbe comunque rimanere quella predominante nel rispetto del carattere pragmatico esperienzale del nostro modello di setting. Descriviamo in sintesi la scansione logica e temporale funzionale alla costruzione di una Classe di Bayes - schematizzata nell'immagine che segue (Figura 1).

6 Si vedano in proposito i documenti al sito http://europa.eu/legislation_summaries/ education_training_youth/lifelong_learning/c11090_en.htm. 


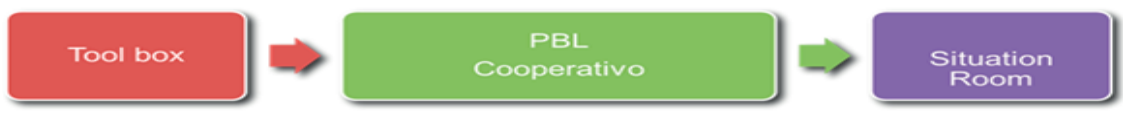

Figura 1. - Lo schema del processo didattico della Classe di Bayes.

1. Tool box. È l'avvio del processo didattico: si tratta di una attività solo apparentemente paragonabile alla didattica frontale tradizionale. In realtà, l'insegnante si vedrà impegnato nel delineare i tratti concettuali essenziali alla comprensione dell'area tematica da affrontare, evidenziando - tanto sincronicamente quanto diacronicamente - le connessioni interdisciplinari e i nuclei tematici fondamentali. Il tutto, facendo emergere le logiche di indagine e le metodologie di ricerca di volta in volta funzionali al contesto in questione. L'applicazione di un tale approccio didattico, già a questo primo step, richiede: una inevitabile una selezione dei contenuti da proporre alla classe e un approccio metodologico e critico all'indagine nei differenti campi del sapere. Tutto ciò per predisporre gli studenti all'acquisizione progressiva di competenze critiche e abilità analitiche, funzionali alle attività di ricerca e di scoperta che caratterizzano la seconda fase del modello didattico che proponiamo. L'insegnante dovrà, infatti, presentare contemporaneamente gli elementi base delle questioni in gioco e la metodologia attraverso la quale gli studenti impareranno a fare da soli. Pertanto, in questa fase i discenti verranno messi nelle condizioni di impossessarsi della «cassetta degli attrezzi» per analizzare e affrontare le tematiche introdotte dall'insegnante - una Tool box insieme metodologica e tecnologica.

Le infrastrutture tecnologiche necessarie per questa prima fase del setting sono: (1) una connessione wifi a banda larga (che copra tutte le classi dell'istituto scolastico); (2) un notebook per l'insegnante; (3) un video proiettore (eventualmente interattivo) o una lavagna interattiva multimediale (LIM); (4) un ambiente virtuale per l'apprendimento (Learning Managment Sistem / Virtual Learning Environment) che permetta di gestire i contenuti digitali e le attività didattiche che si svolgeranno online; (5) device digitali (tablet, notebook) destinati al lavoro attivo degli studenti.

È fondamentale, quindi, che la spiegazione dei «nuclei fondanti» delle singole discipline rispetti gli stili di apprendimento multicodicali degli studenti, utilizzando di volta in volta il codice multimendiale più appropriato. Per fare ciò l'insegnante dovrà ricavare dalle basi dati online (libri digitali) degli editori e/o dai materiali disponibili in rete i contenuti digitali (audio, video, infografiche, ecc.) o da libri e altre fonti cartacee (che andranno digitalizzati per essere inseriti nell'VLE) più utili alla rappresentazione del tema trattato. Tali contenuti potranno essere attinti sia dalle piattaforme che gli 
editori hanno l'obbligo predisporre come componente strutturale dei «libri digitali» (Ferri, 2012), sia da canali Internet quali YouTube, Slideshare, Kahn Accademy, TED, Google Books, Wikipedia, ecc. Oppure, da librerie e biblioteche. Inoltre, tutti i contenuti che l'insegnante avrà ritenuto necessari e sufficienti a questa fase dovranno rimanere disponibili per gli studenti all'interno di una classe virtuale (LCMS/VLE).

Nella nostra ipotesi di «tempo scuola» questa attività formativa - la Tool box, appunto - non dovrebbe mai superare un terzo delle ore che il docente ha stabilito di dedicare a questa o quell'area tematica.

2. Problem solving cooperativo. Questa fase rappresenta il momento centrale della nostra proposta. Gli studenti saranno organizzati dall'insegnante in piccoli gruppi e abilitati a lavorare all'interno di una classe virtuale (LCMS/ VLE). Quindi, sulla base di una scelta di e-tivieties (attività cooperative online) ${ }^{7}$ tra quelle loro prospettate dal docente, dovranno approfondire, analizzare ed eventualmente risolvere i problemi emersi durante la Tool box.

In questa seconda fase, gli studenti agiranno come piccoli ricercatori, sostituendo lo studio tipicamente concepito come sforzo mnemonico con un'indagine razionale modellata - come si vedrà (par. 3) - sulla logica della scoperta scientifica e implementata dagli strumenti di simulazione digitale dell'esperienza e/o di esplorazione e documentazione di fenomeni reali all'interno o all'esterno della scuola.

Durante questa porzione del «tempo scuola» l'insegnante assumerà quindi una funzione di supporto, di scaffolding e di tutoring. Vestendo i panni di un direttore di una serie di piccoli gruppi di ricerca (i gruppi di lavoro), il docente dovrà pertanto affiancare e sostenere i suoi «ricercatori» nella loro attività indagine e revisione razionale delle varie ipotesi ed evidenze di volta in volta emerse dal lavoro dei gruppi. Nello specifico, si tratterà soprattutto di illustrare in pratica (1) le modalità di formulazione di un'ipotesi di ricerca e (2) come si procede, problema per problema, alla sua revisione (corroborazione o confutazione) logica e/o empirica attraverso un'applicazione "qualitativa» della cosiddetta razionalità bayesiana. Non è necessario, a tale scopo, che gli studenti (e i docenti) conoscano il teorema di Bayes e la sua dimostrazione, ma è sufficiente l'apprendimento sul campo della sua pratica euristica (si veda par. 3).

7 Secondo la definizione di Gilly Salmon, il termine identifica un framework di attività finalizzate alla comprensione o all'approfondimento di un tema in modo dinamico e interattivo. Le e-tivities sono, infatti, basate su un intenso scambio e sul dialogo riflessivo tra gli studenti e i docenti che si può svolgere sia all'interno della classe ma soprattutto attraverso un ambiente virtuale per l'apprendimento. Come sostiene Salmon (2002): «e-tivities are designed to engage online students in meaningful work that captures their imagination and challenges them to grow». 
La nostra ipotesi, dunque, è che sia possibile riplasmare lo stesso concetto di classe, nei termini di gruppi di lavoro bayesiani, resi possibili oggi anche grazie all'infrastrutturazione tecnologica del lavoro in aula e nell'extrascuola. Con il termine "gruppi di lavoro bayesiani» (si veda par. 3) pensiamo a gruppi cooperativi in presenza e online che siano in grado di imparare scoprendo insieme e, al contempo, massimizzando il valore della creatività soggettiva e del talento individuale di ciascun membro del gruppo medesimo. Questa specifica caratteristica, implicita nella logica bayesiana, nella sua estensione didattica rappresenta un potente mezzo di valorizzazione dell'atteggiamento critico e della capacità degli studenti di impostare e risolvere problemi ricorrendo non solo alle nozioni apprese in classe, ma anche valorizzando e mettendo al servizio del gruppo di ricerca specifiche attitudini e talenti individuali (personalizzazione dell'apprendimento).

Inoltre, tale strategia di cooperazione razionale - che trasforma l'aula nella Classe di Bayes - trova il suo "naturale» prolungamento nella capacità che le protesi cognitive e digitali (Moriggi \& Nicoletti, 2009) hanno di favorire la simulazione, la rappresentazione, la gestione condivisa e la disseminazione della conoscenza e dei saperi (Gee, 2007; Parisi, 2001).

Il Problem solving cooperativo prevede l'utilizzo dei notebook o dei tablet previsti nella Tool box per il lavoro dei gruppi bayesiani.

3. Situation room. In questa terza fase - come un primo ministro consulta i suoi esperti per gestire uno stato di crisi - l'insegnate allestisce di fatto una Situation room digitalmente aumentata (Wenger, 1998) per l'analisi e la valutazione delle conoscenze. Ovvero, un momento di discussione che condivide e approfondisce i risultati del lavoro dei singoli team di ricerca, anche attraverso l'utilizzo delle fonti disponibili online come elemento di critica e controllo dei risultati e delle congetture degli altri gruppi. È la fase più dialettica della nostra proposta di setting didattico, quella in cui vengono resi "pubblici» i prodotti dei lavori cooperativi.

Gli esiti dei gruppi si rendono, pertanto, disponibili alla critica e alle potenziali obiezioni degli altri «ricercatori», i quali - a loro volta impegnati su ricerche affini e contigue - hanno tutto l'interesse a capire, mettendo alla prova con richieste di chiarimento e osservazioni nel merito, evidenze e conclusioni esposte dai "colleghi». Anche perché i metodi e il lavoro degli altri gruppi potrebbero utilmente integrare o approfondire il proprio. In questo modo, al di là dell'acquisizione di competenze tematiche e contenutistiche, gli studenti si abituano (1) al lavoro di gruppo; (2) all'onestà intellettuale di sottoporre al controllo pubblico le proprie idee e congetture sul mondo e sui saperi; (3) a chiedere conto delle ragioni altrui, esercitando come un diritto/ dovere il pensiero critico nell'interesse proprio e collettivo. 
Questo processo di confronto pubblico e di revisione razionale dei risultati è di fatto reso possibile dall'opportunità di lavorare simultaneamente all'interno di una classe reale e di un ambiente virtuale per l'apprendimento (LCMS/VLE). Un tale ambiente, pensato per la gestione condivisa della conoscenza (Knowldge Management) e per il supporto alla conduzione del processo didattico, rende infatti praticabile una serie di operazioni irrealizzabili nei modi sopra indicati all'interno di un contesto esclusivamente analogico. Più nel dettaglio, l'interazione reale/virtuale all'interno di un contesto formativo ripensato sul modello della Classe di Bayes apre un nuovo orizzonte di opportunità oltre che sul fronte della didattica anche su quello della valutazione. Da un punto di vista didattico:

1. L'utilizzo di Internet come piattaforma di lavoro e condivisione consente, anche durante la Situation room, l'accesso e l'utilizzo diretto da parte degli studenti a una quantità di nozioni e informazioni impensabile in un setting gutemberghiano non abilitato dalle tecnologie.

2. L'elaborazione critica all'interno della classe virtuale dei materiali selezionati (a integrazione dei contenuti forniti dagli editori) facilita la tracciabilità condivisa della propria ricerca (Diario di Laboratorio) - e dunque non solo consente la visibilità dei risultati ma documenta anche il processo metodologico e creativo che ha permesso al gruppo di raggiungerli.

3. Tale dimensione pubblica della ricerca obbliga di conseguenza anche in questa fase di esposizione/discussione i singoli gruppi a documentare ogni passaggio del loro lavoro. Il che, inoltre, induce a una costante ostensione argomentata delle fonti.

Il fatto che ogni singola ricerca sia immediatamente disponibile (nello spazio e nel tempo) a tutti i membri della Classe di Bayes (docente compreso) agevola la comunicazione dei risultati e costituisce il sedimento di una memoria storica e dinamica delle attività dei gruppi. Tutto ciò rende concretamente praticabile una reale valutazione del processo di apprendimento collettivo e individuale. All'interno di un ambiente virtuale, infatti, l'insegnante è in grado di ricostruire - e quindi di valutare - l'effettivo contributo in termini di impegno, creatività, ideazione, collaborazione, progettazione, argomentazione, esposizione e capacità dialettica di ogni singolo «ricercatore».

Definite le tre fasi costitutive della Classe di Bayes e le loro caratteristiche operative, si tratta a questo punto di approfondire i nuclei epistemologici che la sottendono, accennando al contempo alle potenziali ricadute culturali e sociali eccedenti lo specifico contesto della didattica abilitata dalle tecnologie. Il che indurrà anche a ripensare al ruolo e alla rilevanza della scuola come strumento per la realizzazione di una cittadinanza attiva (Mennarini, 2009) ${ }^{8}$.

8 La cittadinanza attiva è la partecipazione del cittadino alla vita civile del suo Paese, onorando i propri doveri e conoscendo ed esigendo i diritti propri e quelli altrui. Il concetto è 


\section{SCUOLA, DEMOCRAZIA E TOLLERANZA: UN'EPISTEMOLOGIA PRAGMATISTA}

In quest'ottica la Classe di Bayes è concepita come un modello didattico/ formativo modulato su una tipologia di cooperazione razionale (abilitata dalle tecnologie) direttamente ispirata alla «logica dell'incerto» - definizione con cui gli epistemologi sono soliti riferirsi al calcolo soggettivistico delle probabilità. Per illustrare questa modalità di rileggere le dinamiche interne alla logica della scoperta scientifica basterà in questa sede soffermarsi su qualche aspetto squisitamente euristico del cosiddetto Teorema di Bayes; e quindi tratteggiare le modalità della sua applicazione al nostro modello di didattica aumentata dalla tecnologia - anche se in termini esclusivamente qualitativi ${ }^{9}$.

Tale formula, conosciuta anche come Teorema della probabilità delle cause, è stata ottenuta nel XVIII secolo da un reverendo protestante con la passione della matematica, Thomas Bayes - il quale l'ha derivata da altri due importanti teoremi: il teorema della probabilità composta e il teorema della probabilità assoluta ${ }^{10}$.

In generale, la "formula» di Bayes costituisce un potente strumento di critica e crescita della conoscenza utile a discriminare tra ipotesi alternative (o in conflitto), attraverso una revisione razionale delle ipotesi stesse - che non trascura intrinsecamente nemmeno quelle (apparentemente!) più stravaganti.

Più precisamente il concetto soggettivistico della probabilità rivela una efficace applicazione alla didattica attiva sopra descritta in quanto fornisce una matrice concettuale attraverso cui correggere o raffinare le nostre ipotesi, e dunque il nostro grado di fiducia in esse - degree of belief -, alla luce di un qualche supplemento di informazione o di indagine. Pertanto, la formula del reverendo mette a disposizione degli studenti e degli insegnanti un approccio metodologico - e, più in generale, un atteggiamento critico - che, come ha puntualmente osservato il fisico Carlo Rovelli (facendo tesoro degli sviluppi tecnici e concettuali consentiti dai contributi del matematico italiano Bru-

stato spesso accostato - e a volte addirittura fatto coincidere - col volontariato e l'associazionismo. Tuttavia, per quanto queste attività abbiano un ruolo determinante nell'esercizio e nella diffusione di una cittadinanza attiva - oltre che nella sua incubazione culturale (Menarini, 2009) - gli autori del presente articolo non ritengono che possano ipso facto comprendere ed esaurire le molteplici valenze culturali e gli stili cognitivi sottesi al concetto in questione.

9 Allo sviluppo tecnico e alla formalizzazione di questa metodologia didattica e di valutazione sarà dedicato un nostro prossimo articolo.

10 Per un approfondimento di temi e problemi della logica bayesiana si rimanda a Hacking, 2005. 
no de Finetti ${ }^{11}$ ) si configura come "gestione oculata e razionale della nostra ignoranza» (Rovelli, 2013).

Una osservazione più ravvicinata del Teorema di Bayes (presentato nella sua formulazione più semplificata) consentirà di cogliere per lo meno alcuni degli aspetti fondamentali della pratica scientifica a esso sottesa, decisivi allo scopo di ridefinire le coordinate base e gli obiettivi di massima della nostra proposta didattica.

Sia $\mathrm{H}_{0}$ l'ipotesi di partenza ed $\mathrm{E}$ il dato empirico osservato:

$$
\mathrm{P}\left(\mathrm{H}_{0} / \mathrm{E}\right)=\frac{\mathrm{P}\left(\mathrm{E} / \mathrm{H}_{0}\right) \mathrm{P}\left(\mathrm{H}_{0}\right)}{\mathrm{P}(\mathrm{E})}
$$

- $\mathrm{P}\left(\mathrm{H}_{0}\right)$ è la probabilità a priori di $\mathrm{H}_{0}$. Si tenga conto che non è rilevante la provenienza dell'ipotesi di partenza $\mathrm{H}_{0}$. Essa, per definizione, costituisce la congettura formulata prima di ogni controllo empirico e osservativo.

- $\mathrm{P}\left(\mathrm{E} / \mathrm{H}_{0}\right)$ : è detta funzione di verosimiglianza e su di essa cui si fonda l'inferenza classica (o frequentista) in statistica.

- $\mathrm{P}(\mathrm{E})$ : probabilità a priori di $\mathrm{E}$ (detta costante di normalizzazione).

- $\mathrm{P}\left(\mathrm{H}_{0} / \mathrm{E}\right)$ è la probabilità a posteriori di $\mathrm{H}_{0}$ dato $\mathrm{E}$.

- $\mathrm{P}\left(\mathrm{E} / \mathrm{H}_{0}\right) / \mathrm{P}(\mathrm{E})$ : è detto «fattore di scala» e consente di ponderare l'impatto che l'osservazione di $\mathrm{E}$ ha sul grado di confidenza del ricercatore nell'ipotesi iniziale, rappresentato a sua volta dalla probabilità a priori $\mathrm{P}\left(\mathrm{H}_{0}\right)$.

Il che, come sopra accennato, innesca una prassi di analisi e revisione continue circa l'attendibilità delle proprie ricerche alla luce di successive corroborazioni o smentite delle ipotesi «sotto controllo».

La probabilità (credenza) a posteriori, di conseguenza, combina le convinzioni che il ricercatore ha a priori con quelle derivanti dall'osservazione e dall'analisi del dato empirico acquisito nelle fasi di indagini e approfondimento. Da questo punto di vista, pare opportuno sottolineare almeno due aspetti dell'approccio bayesiano, rilevanti proprio in ragione della sua potenziale ricaduta metodologica e culturale nel più circoscritto mondo delle metodologie didattiche.

Tale prospettiva euristica assume che non sia possibile assegnare agli eventi/ipotesi probabilità oggettive (scetticismo bayesiano). In altre parole, le probabilità devono essere intese come gradi soggettivi di fiducia del ricercatore (degrees of belief). Il che è di per sé sufficiente per lasciare intendere come l'epistemologia bayesiana consenta di tener conto di un dato di fatto non trascurabile, specie all'interno di gruppo di ricerca (o di studio): è

11 Si veda, per esempio, de Finetti, 2006. 
sommamente improbabile che individui tra loro diversi, relativamente a un tema o a un problema dato, muovano dallo stesso grado di confidenza soggettiva iniziale. Ovvero, molto difficilmente i soggetti in questione sarebbero disposti a scommettere su una stessa ipotesi (di ricerca o di lavoro) e con lo stesso grado di fiducia nei suoi confronti. È proprio l'adozione di una pratica euristica come quella bayesiana che può educare a imparare dall'esperienza. A fronte di dati e nozioni acquisite nelle fasi si ricerca, infatti, si apprende come rivedere (corroborare, correggere o confutare) il grado di fiducia nelle ipotesi su cui si era disposti a scommettere in partenza. Il che consente e agevola una selezione ragionata del ventaglio di opzioni da cui il gruppo (di ricerca o di lavoro) aveva preso le mosse. Del resto, per quanto i soggetti coinvolti nel gruppo possano essere partiti con gradi di fiducia soggettiva (a priori) molto differenti, un numero sufficiente di osservazioni (e revisioni) guida gli attori della ricerca verso probabilità a posteriori tra loro sempre più vicine.

Il secondo aspetto, strettamente connesso al primo, consiste nel fatto che i ricercatori (gli studenti) non possano rigettare a priori le ipotesi alternative (o avversarie) alle loro. Non si tratta semplicemente di una tolleranza etica, ma anzitutto epistemologica. Nessun componente di un gruppo (di lavoro o di ricerca), infatti, può tecnicamente escludere o scartare alcuna ipotesi in assenza di dati o argomenti sufficienti. La capacità di gestione da parte dell'insegnante dei gruppi di lavoro durante il Problem solving cooperativo consiste proprio nel sorvegliare e accompagnare tali unità di ricerca nei processi di revisione razionale delle ipotesi, abituando gli attori di tale apprendimento attivo a un confronto basato sul suddetto principio di tolleranza epistemologica. E da un punto di vista euristico (oltre che etico), tale pratica induce - nell'esercizio di apprendimento critico - a ottimizzare all'interno delle dinamiche del gruppo i talenti dei singoli, più o meno eccentrici che siano. Inoltre, una buona conduzione da parte del docente di questa fase del lavoro dei gruppi (e, dunque, dei singoli all'interno dei gruppi) è la condizione necessaria all'avvio del pubblico processo di esposizione e confronto dei risultati ottenuti proprio nella Situation room.

Sono questi i processi e le fasi operative che, tra l'altro, consentono di mettere in pratica in un contesto didattico le dinamiche proprie della logica della scoperta scientifica. La Situation room, infatti, ricalca a suo modo quel consesso di esperti che oggi chiamiamo comunità scientifica e che al tempo di Galileo Galilei era detta (si badi alle parole!) Repubblica delle Lettere. Si tratta di una società (popperianamente) «aperta» e di "pari» in cui (1) la conoscenza è la risultante di una libera cooperazione e da intendersi nei termini di un patrimonio pubblico e (2) l'opzione migliore non è mai imposta in ragione di una qualche maggioranza, ma sempre valutata sulla base della attendibilità teorica e/o empirica delle tesi esposte. In questo modo, come si 
accennava più sopra, nella Situation room gli insegnanti potranno valutare il lavoro realizzato dai team di giovani scienziati (gli studenti) anche attraverso la mediazione e il supporto delle tecnologie.

Tale ipotesi di setting, pertanto, si prefigge di mettere in evidenza la concreta opportunità di un prossimo «ritorno al futuro" per il sistema scolastico e formativo italiano. Dal momento che consentirebbe, attraverso le tecnologie digitali dell'apprendimento, di concretizzare su larga scala la utopia positiva del learning by doing di John Dewey - rivista però alla luce di un approccio logico-metodologico in grado di rendere sostenibili e produttivi i cambiamenti resi necessari dalla transizione al digitale dell'istituzione scolastica. In particolare, questa proposta tenderebbe a rivitalizzare la realtà molto spesso «autocentrata» e tradizionalista della didattica all'interno delle agenzie formative italiane. Proporre tale ipotesi di «riforma» a tutti i soggetti coinvolti nel sistema-formazione (studenti, docenti, dirigenti scolastici, genitori) costituisce l'obiettivo di questa prima esposizione sintetica e forzatamente enunciativa di un più ampio lavoro di ricerca in corso d'opera.

\section{Conclusioni}

Sulla base di quanto osservato, pare opportuno ribadire due aspetti legati - e tra loro connessi - a un percorso ragionato di digitalizzazione della didattica. $\mathrm{Se}$, infatti, da un lato è sempre più evidente come un setting gutemberghiano si mostri insufficiente, per non dire incompatibile con gli stili di apprendimento sviluppati dai nativi digitali; d'altro canto, pare evidente che il progetto di digitalizzazione della scuola (e dell'insegnamento) non possa esaurirsi nei termini di un aggiornamento informatico dell'istituzione e del personale docente. Proprio alla luce di tale consapevolezza - ovvero della necessità di ripensare e riplasmare le dinamiche (oltre che gli spazi) dell'apprendimento, ci è parso di cogliere in quella che poteva sembrare - e che per molti versi di fatto è - una fase critica della scuola, una grande opportunità. La nuova scuola digitalmente aumentata, nello scardinare l'assetto tradizionale della lezione frontale, apre un orizzonte fisico e concettuale funzionale a una effettiva riabilitazione della didattica attiva. E non solo di una generica ripresa di un antico, per quanto opportuno, imparare facendo. L'esternalizzazione delle fonti del sapere nel contesto allargato della rete richiede infatti sempre più che quel fare di antica memoria venga riproposto, ma ridefinito sulla base di quella metodologia che, come nessun'altra, consente - come si diceva - una gestione razionale della nostra ignoranza. Ovvero, in altre parole, l'acquisizione di un metodo di analisi e di critica mutuabile solo dalla logica della ricerca 
scientifica. Inoltre, la scelta di ricorrere alla logica dell'incerto come prospettiva epistemologica attraverso cui calare le dinamiche della ricerca nella pratica della didattica va cercata, di certo, nell'efficacia di tale approccio euristico, ma anche (anzi, soprattutto) nelle sue potenzialità di definire una ricerca cooperativa in cui i vantaggi del lavoro di gruppo non rischiano di sacrificare le qualità dei singoli. Ė nostra convinzione che proprio all'interno di queste dinamiche della ricerca potranno germogliare le concrete premesse di una cittadinanza attiva non riducibile alle buone intenzioni del volontariato e dell'associazionismo.

\section{RIFERIMENTI BIBLIOGRAFICI}

Castells, M. (1996). The information age: Economy, society and culture, Vol. I: The rise of network society. Malden, MA: Blackwell (trad. it., Milano: Università Bocconi).

Castells, M., \& Himanen, P. (2002). The information society and the welfare state. The finnish model. New York: Oxford University Press (trad. it., Milano: Guerini e Associati).

Cope, B., \& Kalantzis, M. (Eds.). (2000). Multiliteracies: Literacy learning and the design of social futures. London: Routledge.

Crook, Ch. (2008b). Web 2.0 technologies for learning: The current landscape Opportunities, challenges and tensions. http://partners.becta.org.uk/index. php?section=rh\&\&catcode=_re_rp_02\&rid=15878 (consulted 13/11/2014).

de Finetti, B. (2006). Linvenzione della verità. Milano: Raffaello Cortina.

Dewey, J. (1938). Experience and education. New York: Kappa Delta Pi, International Honor Society in Education (trad. it., Milano: Raffaello Cortina).

Ferri, P. (2008). La scuola digitale. Come le nuove tecnologie cambiano la formazione. Milano: Bruno Mondadori.

Ferri, P. (2011). Nativi digitali. Milano: Bruno Mondadori.

Ferri, P. (2012). Scuola, il governo mette in difficoltà famiglie ed editori. http://www. agendadigitale.eu/egov/115_scuola-il-governo-mette-in-difficolta-famigliee-editori.htm (consulted 13/11/2014).

Ferri, P. (2013). La scuola 2.0. Verso una didattica aumentata dalle tecnologie. Parma: Spaggiari.

Ferri, P. (2014). I nuovi bambini. Come ducare i figli all'uso delle tecnologie, senza diffidenze e paure. Milano: Rizzoli.

Ferri, P., \& Mantovani, S. (2006). Bambini e computer. Alla scoperta delle nuove tecnologie a scuola e in famiglia. Milano: RCS Etas. 
Ferri, P., \& Mantovani, S. (2008). Digital kids. Come comunicano e apprendo in nativi digitali e come potrebbero farlo genitori e insegnanti. Milano: RCS Etas.

Ferri, P., Mizzella, S., \& Scenini, F. (2009). I nuovi media e il web 2.0. Comunicazione, formazione ed economia nella società digitale. Milano Guerini e Associati.

Gasser, U., \& Palfrey, J. (2008). Born digital-Connecting with a global generation of digital natives. New York: Perseus.

Gee, J. P. (2007). What video games have to teach us about learning and literacy, revised and updated. Basingstoke: Palgrave Macmillan (trad. it., Milano: Raffaello Cortina).

Hacking, I. (2001). An introduction to probability and inductive logic. Cambridge: Cambridge University Press (trad. it., Milano: il Saggiatore).

Himanen, P. (2001). The hacker ethic and the spirit of the information age. New York: Random House (trad. it., Milano: Feltrinelli).

Jenkins, H. (2006). Convergence culture: Where old and new media collide. New York: NY University Press.

Jenkins, H. (2009). Confronting the challenges of participatory culture. Media education for the 21st century. Cambridge, MA: MIT Press.

Jonassen, D. H. (2005). Modeling with technology: Mindtools for conceptual change (3rd ed.). Columbus, OH: Prentice-Hall.

Livingstone, S., Haddon, L., Görzic, A., \& Ólafsson, K. (2011). EU kids on-line. Final report, London: LSE. http://eprints.lse.ac.uk/39351/ (consulted 11/11/ 2014).

Mack, A. M., \& Palley, W. (2012). Gen Z: Digital in their Dna. http://www.jwtintelligence.com/wp-content/uploads/2012/04/F_INTERNAL_Gen_Z_0418122. pdf. (consulted 11/11/2014).

Mannarini, T. (2009). Cittadinanza attiva. Psicologia sociale della partecipazione pubblica. Bologna: il Mulino.

Moriggi, S. (2014). Connessi. Beati quelli che sapranno pensare con le macchine. Milano: San Paolo.

Moriggi, S., \& Nicoletti, G. (2009). Perché la tecnologia ci rende umani. Le riscritture sintetiche e digitali della carne. Milano: Sironi.

Parisi, D. (2001). Simulazioni. La realtà rifatta nel computer. Bologna: il Mulino.

Prensky, M. (2006). Mama don't bother me Mom - I'm learning. New York: Paragon House (trad. it., Perugia: Multiplayer.it).

Prensky, M. (2001). Digital natives, digital immigrants. On the Horizon, 9(5), 1-6.

Prensky, M. (2012). Brain gain: Technology and the quest for digital wisdom. Basingstoke: Pallgrave Macmillan (trad. it., Trento: Erickson).

Pullen, D. L., \& Cole, D. R. (2009). Multiliteracies and technology enhanced education. Social practice and the global classroom. Herschey, PA: IGI Group. 
Rovelli, C. (2013). Sì, no, anzi: probabilmente. Il Sole24Ore - Domenica, 20 Gennaio 2013.

Salmon, G. (2002). E-tivities: The key to active online learning. London: Kogan Page.

Smith, P., Rudd, P., \& Coghlan, M. (2008). Harnessing technology: Schools Survey (2008). http://partners.becta.org.uk/upload-dir/downloads/page_documents/ research/ht_schools_survey08_executive_report.pdf. (consulted 11/11/2014).

Spiro, R. J., Collins, B. P., \& Ramchandran, A. R. (2006). Modes of openness and flexibility in cognitive flexibility hypertext learning environments. In B. Khan (Ed.), Flexible learning (pp. 18-25). Hershey, PA: Information Science.

Wenger, E. C. (1998). Community of practice: Learning, meaning and identity, Cambridge: Cambridge University Press.

\section{RiassunTO}

Il divario tra i nuovi stili di apprendimento dei "nativi digitali» e le strategie e le pratiche didattiche della scuola italiana è ancora molto ampio. Si rende quindi necessario ripensare radicalmente l'impostazione dell'insegnamento e dell'apprendimento almeno per quanto riguarda tre questioni fondamentali: (a) gli spazi fisici dell'istruzione, (b) gli approcci metodologici, (c) le tecnologie coinvolte nel processo di apprendimento. L'articolo ha lo scopo di delineare una possibile proposta per la nuova "educazione aumentata» dalla tecnologia digitale, basata su un approccio socio-costruttivista. L'analisi di dati ed evidenze relativi agli stili comportamentali e cognitivi delle nuove generazioni ci ha condotto a individuare nella logica della scoperta scientifica (nel caso specifico riletta alla luce dell'epistemologia bayesiana) la matrice culturale più consona per sviluppare un modello di didattica aumentato dalle tecnologie. Per esporre la nostra proposta, si è pensato di suddividere l'articolo in tre parti: nella prima descriviamo come e perché il "brain-frame» dei «nativi digitali» $e ̀$ incompatibile con una didattica ancora largamente gutemberghiana. Nella seconda, delineiamo la nostra proposta metodologica e tecnologica che si articola in tre fasi: "Tool box», "Problem solving" cooperativo e "Situation room». La parte terza trae alcune conclusioni riguardi l'impatto di questo nostro approccio sul concetto di cittadinanza democratica.

Parole chiave: Didattica, Digitalizzazione, Epistemologia bayesiana, Scuola italiana, Stili di apprendimento dei nativi digitali. 